\title{
Cifosis funcional y actitud cifótica lumbar en piragüistas adolescentes Functional kyphosis and lumbar kyphosis in adolescent paddlers
}

\author{
*Pedro Ángel López-Miñarro, *Fernando Alacid Cárceles \\ *Universidad de Murcia (España)
}

\begin{abstract}
Resumen: El objetivo de este estudio fue valorar la frecuencia de casos de cifosis funcional y actitud cifótica lumbar en piragüistas adolescentes. A un total de 140 piragüistas (media de edad: 13,67 $\pm 0,61$ años) se les midió, mediante un inclinómetro, la disposición sagital del raquis dorsal y lumbar en bipedestación relajada y al realizar una flexión máxima del tronco en sedentación con rodillas extendidas (test de distancia dedos-planta). Al clasificar los valores angulares en base a las referencias de normalidad, un 63\% de los deportistas tenían una curva dorsal normal en bipedestación, de los cuales un $91,2 \%$ presentaban un morfotipo cifótico leve o moderado en la posición de máxima flexión del tronco. En cuanto al raquis lumbar, el 68,5\% tenía una curva normal en bipedestación, de los cuales el 83,9\% presentaban un morfotipo cifótico en flexión máxima del tronco. En conclusión, existe un alto porcentaje de piragüistas con una disposición normal del raquis en bipedestación, pero con un morfotipo cifótico leve o moderado en flexión máxima del tronco. Es necesario incluir, en el control y seguimiento de estos deportistas, una valoración de la columna que incluya el estudio de la disposición sagital del raquis en flexión máxima del tronco.
\end{abstract}

Palabra clave: raquis, torácico, lumbar, flexión máxima del tronco, bipedestación.

\begin{abstract}
The objective of this study was to evaluate the frequency of functional kyphosis and lumbar kyphosis in adolescent paddlers. A total of 140 paddlers (mean age: 13,67 $\pm 0,61$ years) participated in this study. The sagittal spinal curvatures (thoracic and lumbar curves) in relaxed standing and in maximal trunk flexion with knees extended (sit-and-reach test) were evaluated with an inclinometer. The analysis of angular values with respect to the normality references showed that $63 \%$ of athletes with normal thoracic curve in standing, while in maximal trunk flexion there were many athletes (91.2\%) with a moderate or slight kyphotic posture. With respect to lumbar curve, $68.5 \%$ of the subjects had normal values in standing, while $83.9 \%$ showed lumbar kyphotic postures in maximal trunk flexion. In conclusion, there was a high percentage of paddlers with normal angular values in relaxed standing, although when maximal trunk flexion with knees extended was performed there was a significant increase of kyphotic postures in both thoracic and lumbar curves. For this reason, the evaluation of sagittal spinal curvatures of paddlers is an important variable to include in training planning.
\end{abstract}

Key words: spine, thoracic, lumbar, maximal trunk bending, standing.

\section{Introducción}

El estudio y valoración de la disposición sagital del raquis en deportistas jóvenes es importante porque diversos estudios han encontrado cambios específicos del morfotipo raquídeo según el deporte realizado (Boldori, Da Soldá, \& Marelli, 1999; Uetake \& Ohtsuki, 1998; Wojtys, Ashton-Miller, Huston, \& Moga, 2000), y porque existe una elevada frecuencia de desalineaciones raquídeas en el período puberal y la adolescencia (Ferrer, 1998; Serna, Santonja, \& Pastor, 1996). Estas desalineaciones pueden generar repercusiones (Murray, Weinstein, \& Spratt, 1993) si no se detectan y no se adoptan las medidas adecuadas.

Varios estudios han valorado el morfotipo raquídeo en deportistas, tales como nadadores (Pastor, 2000), gimnastas de rítmica (Kums, Ereline, Gapeyeva, Pääsuke, \& Vain, 2007; Nilsson, Wykman, \& Leanderson, 1993; Martínez, 2004; Ohlén, Wredmark, \& Spandfort, 1989), bailarinas (Gómez, 2007), futbolistas (López, Alburquerque, Quintana, Domínguez, Rubens, \& Calvo, 2005; Sáinz de Baranda etal., 2001; Wodecki, Guigui, Hanotel, Cardinne, \& Deburge, 2002), usuarios de salas de musculación (López-Miñarro, Rodríguez, Santonja, Yuste, \& García, 2007a; López-Miñarro, Yuste, Rodríguez, Santonja, Sáinz de Baranda, \& García, 2007b; López-Miñarro, Rodríguez, Santonja, \& Yuste, 2008d), piragüistas (López-Miñarro,Alacid, Ferragut, \& García, 2008a), remeros (Howell, 1984; Stuchfield \& Coleman, 2006) esquiadores (Alricsson \& Werner, 2006) y luchadores (Rajabi, Doherty, Goodarzi, \& Hemayattalab, 2008), encontrando adaptaciones específicas del morfotipo raquídeo al deporte practicado.

La mayoría de estos estudios han analizado el morfotipo raquídeo en bipedestación. Sin embargo, esta posición sólo es una de las que se pueden adoptar en la vida cotidiana y en la práctica físico-deportiva.

Fecha recepción: 02-09-09 - Fecha envío revisores: 23-09-09 - Fecha de aceptación: 01-11-09 Correspondencia: Pedro A. López-Miñarro.

Facultad de Educación. Universidad de Murcia.

Campus Universitario de Espinardo, 30100 (Murcia)

E-mail: palopez@um.es
Diversos autores han resaltado la importancia de incluir, además, el estudio del morfotipo raquídeo en la posición de flexión máxima del tronco (Santonja, 1996; Stagnara, 1987; Serna etal., 1996). En esta línea, algunos estudios que analizan el morfotipo raquídeo en deportistas, han incluido el análisis de la disposición sagital del raquis en flexión máxima del tronco (Gómez, 2007; López-Miñarro \& Alacid, 2009; Martínez, 2004; Pastor, 2000; Sáinz de Baranda et al., 2001).

$\mathrm{Al}$ realizar la exploración de la disposición sagital del raquis, se puede encontrar una correcta disposición del raquis en bipedestación, pero con un notable incremento de la curvatura dorsal en la posición de flexión máxima del tronco, lo que se ha denominado «cifosis funcional» (Santonja, 1996; Santonja, Pastor, \& Serna, 2000; Santonja, Rodríguez, Sáinz de Baranda, \& López-Miñarro, 2004). Otro problema frecuente es la inversión del raquis lumbar en flexión máxima del tronco, partiendo de una lordosis normal en bipedestación (actitud cifótica lumbar) (Santonja \& Martínez, 1992; Santonja et al., 2000), o de una hiperlordosis lumbar (hipermovilidad lumbar) (Somhegyi \& Ratko, 1993).

La detección de estos morfotipos raquídeos alterados en flexión máxima del tronco adquiere una gran importancia durante el crecimiento, ya que son indicativos de una tendencia a la estructuración de una desalineación raquídea (Santonja etal., 2000; Swärd, 1992), y aumentan el riesgo de repercusiones raquídeas en las estructuras discales, óseas y ligamentosas al aumentar las cargas compresivas, de cizalla anterior y la presión intradiscal (Briggs et al., 2007; Callaghan \& McGill, 2001; McGill, 2002; Polga etal., 2004; Solomonow, 2004; Wilke, Neef, Caimi, Hoogland, \& Claes, 1999).

Debido a las repercusiones asociadas a la cifosis funcional y actitud cifótica lumbar, es preciso conocer su frecuencia en población deportista joven, para instaurar, a continuación, aquellas medidas terapéuticas que permitan mejorar el morfotipo raquídeo y disminuir el riesgo de repercusiones. En este sentido, los objetivos de este estudio fueron: 1) determinar la frecuencia de cifosis funcional en piragüistas adolescentes; y 2) determinar la frecuencia de la actitud cifótica lumbar en piragüistas adolescentes. 


\section{Material y método}

\subsection{Muestra.}

Un total de 140 piragüistas varones, entre 13 y 15 años participaron en el estudio (edad: 13,67 $\pm 0,61$ años; talla: $167,15 \pm 7,67 \mathrm{~cm}$; masa: $61,97 \pm 9,03 \mathrm{~kg}$ ). Los criterios de inclusión fueron: entrenar al menos 5 días a la semana, con una duración superior a 60 minutos por sesión, y desde al menos 2 años de forma continuada. Los criterios de exclusión fueron: tener dolor lumbar o torácico en el mes previo a las mediciones, haber sido sometido a cirugía en la columna vertebral, pelvis o miembros inferiores, o haber tenido algún tipo de lesión aguda o crónica en la columna vertebral y/o musculatura isquiosural en los últimos 6 meses.

\subsection{Procedimiento.}

El estudio fue aprobado por el Comité Ético y de Investigación de la Universidad de Murcia. Los padres y los deportistas fueron informados de los objetivos y métodos del estudio y se obtuvo un consentimiento informado.

Previamente a la medición de la disposición sagital del raquis, las apófisis espinosas de la primera vértebra torácica (T1), duodécima vértebra torácica (T12) y quinta vértebra lumbar(L5) fueron localizadas ymarcadas por un investigador con 10 años de experiencia en la exploración del raquis (fiabilidad intraobservador $>0.93$ para las mediciones en bipedestación $y>0.89$ para las mediciones en flexión máxima del tronco), en base al protocolo utilizado previamente por López-Miñarro et al. (2009). A continuación, se midió de forma aleatoria y en dos ocasiones, mediante un inclinómetro Unilevel (ISOMED, Inc., Pórtland, OR), las curvas dorsal y lumbar en bipedestación relajada y en flexión máxima del tronco con rodillas extendidas (test de distancia dedos-planta). Entre cada medición hubo un descanso de 5 minutos. Para el análisis de datos se utilizó el valor medio de ambas mediciones.

Para medir las curvas raquídeas en bipedestación, el deportista se situaba de pie, con los pies separados a una distancia equivalente a su anchura coxofemoral, los brazos pegados en sus costados y relajados, con la mirada al frente. Una vez colocado, se procedía a la medición, permaneciendo el deportista sin moverse. Se utilizó el protocolo de medición descrito previamente por Mellin (1986) y utilizado posteriormente en deportistas jóvenes por Pastor (2000). Para medir la cifosis torácica el inclinómetro se colocó al inicio de la curvatura torácica, en el lugar donde se obtenía el mayor valor angular de la misma, generalmente coincidente con T1-T3 (Figura 1a), situándose en esta posición a $0^{\circ} \mathrm{y}$, a continuación, se contorneaba el perfil del raquis hasta la zona donde se obtenía el mayor valor angular (final de la curvatura cifótica), generalmente coincidente con T12-L1 (transición lumbosacra) (Figura 1b), obteniendo el grado de la cifosis torácica. Para medir la lordosis lumbar, en el punto donde se determinó el ángulo de la cifosis torácica, se niveló el inclinómetro a $0^{\circ}$ y se colocó, a continuación, al final de la curvatura lordótica, en el punto donde se obtenía el mayor valor angular de la curva, normalmente coincidente con L5-S1 (Figura 1c).

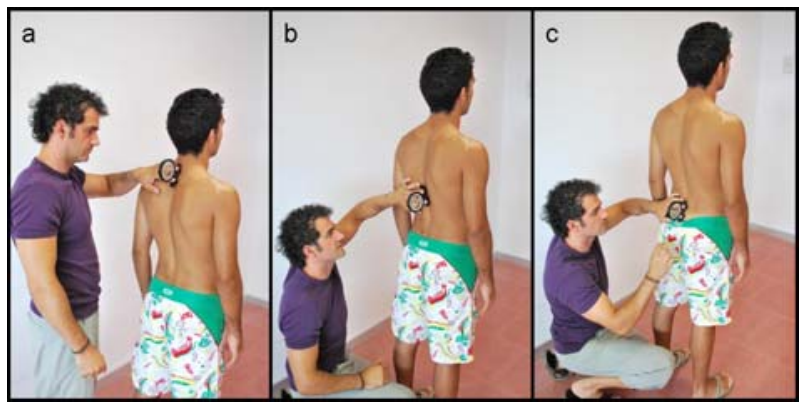

Figura 1. Colocación del inclinómetro en la medición del bipedestación relajada al inicio de la curvatura torácica (a), en el lugar donde se obtiene el mayor valor angular (b), y al final de la curva lumbar (c).
Para categorizar a los deportistas en base al valor angular de la cifosis dorsal se utilizaron las referencias aportadas por Santonja (1993) (Tabla 1). Los valores de referencia de la curva lumbar que se utilizaron para categorizar a los sujetos fueron los propuestos por Pastor (2000) (Tabla 1). Los valores negativos corresponden a una curva lumbar de concavidad posterior (lordosis), mientras que valores positivos indican concavidad anterior (inversión).

Las curvaturas torácica y lumbar fueron también valoradas al realizar una flexión máxima del tronco en sedentación con las rodillas extendidas (test de distancia dedos-planta) siguiendo el protocolo de medición y las técnicas descritas por Mellin (1986) y utilizado posteriormente por otros estudios (Martínez, 2004; Miñarro, Andújar, García, \& Toro, 2007; Pastor, 2000; Rodríguez, 1998). Puesto que en esta posición el raquis adopta una postura de cifosis total (cifosis torácica y lumbar), se tomó como criterio de la transición tóraco-lumbar, la duodécima vértebra torácica.

Para realizar el test, el deportista se situó en sedentación, con las rodillas extendidas y los pies separados a la anchura de sus caderas. Las plantas de los pies se colocaron perpendiculares al suelo, en contacto con un cajón de medición (ACCUFLEX TESTER I). En esta posición, se le solicitó al deportista que realizara una flexión máxima del tronco con rodillas y brazos extendidos. Un investigador se encargó de fijar las rodillas en extensión durante el test. Las palmas de las manos se tenían que deslizar sobre el cajón, hasta alcanzar la máxima distancia posible, manteniendo la posición durante 5 segundos. $\mathrm{Al}$ alcanzar la máxima distancia, se colocó el inclinómetro al inicio de la curvatura torácica, a nivel de T1 (Figura 2a), situándolo a $0^{\circ}$. A continuación se colocó el inclinómetro centrado en la marca de T12 (Figura 2b), obteniendo el valor angular de la curva torácica. En esta posición se volvió a situar el inclinómetro $\mathrm{a} 0^{\circ} \mathrm{y}$ a continuación se colocó al final de la curva lumbar, justo debajo de la marca realizada en L5 (Figura 2c), por lo que el inclinómetro quedaba situado a nivel de la primera vértebra sacra.



Figura 2. Colocación del inclinómetro en el test de distancia dedos-planta al inicio de la curvatura torácica (a), a nivel de T12 (b), y al final de la curvatura lumbar (c).

Para clasificar los valores angulares de las curvas dorsal y lumbar se utilizaron las referencias aportadas por Pastor (2000) (Tabla 1).

\subsection{Análisis estadístico.}

Para verificar la normalidad de las variables se utilizó la prueba de Kolmogorov Smirnov, que evidenció una distribución normal de las mismas. Para comparar los valores angulares de la curva dorsal y lumbar entre la posición de bipedestación y la posición de flexión máxima del tronco en el test de distancia dedos-planta se realizó una prueba t de Student para muestras apareadas. Se realizó un análisis descriptivo de cada una de las variables con la obtención de la distribución de frecuencias en base a las referencias de normalidad. El nivel de significación estadística se estableció en un valor de $p<0.05$. Todos los datos fueron analizados usando el paquete estadístico SPSS versión 15,0. 


\section{Resultados}

Los valores medios de la curva lumbar y dorsal en bipedestación y en el test de distancia dedos-planta se presentan en la figura 3. Se encontraron diferencias significativas $(p<0.001)$ en los valores angulares de ambas curvas raquídeas entre la posición de bipedestación y la máxima flexión del tronco.

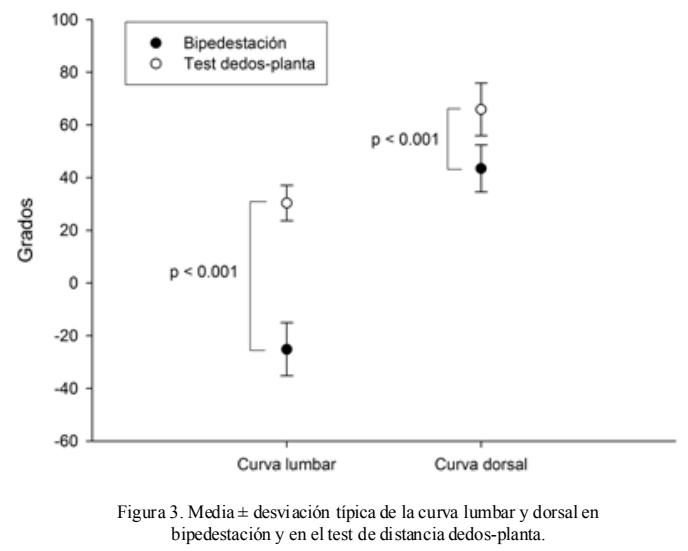

En la tabla 2 se presenta la distribución de casos, en función de las referencias de normalidad de la curva dorsal, para la posición de bipedestación y flexión máxima del tronco. Un $37 \%$ de los deportistas tenían una hipercifosis dorsal en bipedestación. La mayoría de estos adoptaban un morfotipo cifótico en el test de distancia dedos-planta. Del 63\% de deportistas con una cifosis dorsal normal en bipedestación, la mayoría de ellos, el 91,2\%, tenían un morfotipo dorsal cifótico leve o moderado en el test de distancia dedos-planta (cifosis funcional) (Tabla 2).

En cuanto a la curva lumbar, hubo un predominio de posturas normales en bipedestación, seguido de posturas de rectificación. Los casos de hiperlordosis lumbar fueron más reducidos (Tabla 3). Del $68,5 \%$ de deportistas con una lordosis normal en bipedestación, el $83,9 \%$ tenían un morfotipo cifótico lumbar leve o moderado en el test de distancia dedos-planta (actitud cifótica lumbar). De los 8 casos de hiperlordosis lumbar en bipedestación, 6 de ellos tenían un morfotipo cifótico lumbar en el test de distancia dedos-planta (hipermovilidad lumbar).

\begin{tabular}{clcc}
\hline Tabla 1. Referencias de normalidad de las curvas dorsal y lumbar en bipedestación y flexión máxima del tronco \\
con rodillas extendidas.
\end{tabular}

\section{Discusión}

El objetivo principal de este estudio fue describir la frecuencia de casos de cifosis funcional y actitud cifótica lumbar en deportistas jóvenes. Con la exploración sagital del raquis en bipedestación, encontramos un predominio de deportistas con un morfotipo raquídeo normal. No obstante, al valorar el comportamiento del raquis en una posición de flexión máxima del tronco con las rodillas extendidas, los resultados muestran un alto porcentaje de morfotipos alterados, con la gran mayoría de los deportistas adoptando morfotipos cifóticos leves o moderados en la curva dorsal y/o lumbar. Estas posturas cifóticas y/o de inversión lumbar mantenidas o repetitivas en el raquis, aún en proceso de maduración, pueden producir alteraciones en los núcleos de crecimiento de las vértebras (Ashton-Miller, 2004), lo que aumenta la importancia de medir la postura de flexión máxima del tronco cuando se realiza una valoración del morfotipo raquídeo en deportistas.

En la adolescencia, los cuerpos vertebrales van adquiriendo su morfología definitiva y son susceptibles de deformidades estructurales que pueden ser permanentes. Los períodos de crecimiento longitudinal más rápidos son momentos de alta vulnerabilidad para la columna vertebral, por lo que es necesario realizar una adecuada exploración del raquis como elemento de control (Swärd, 1992). Si un morfotipo cifótico no es detectado y, por tanto, no se inicia una intervención terapéutica, puede transformarse, a la larga, en una deformidad estructurada (Ventura, 1986; Domisse, 1990). La edad de los deportistas evaluados en este estudio supone que sus núcleos de crecimiento vertebral aún siguen activos y, por tanto, una postura inadecuada del raquis podría generar alteraciones que afectarían a su calidad de vida, así como a su trayectoria deportiva. En la adolescencia, las alteraciones de la disposición sagital del raquis son más frecuentes que en la infancia y, por lo general, más graves, dada su tendencia a convertirse en deformidades verdaderas o estructuradas.

En este sentido, Pastor (2000) encontró mayor presencia de acuñamientos vertebrales en la transición tóraco-lumbar de aquellos nadadores que presentaban mayor flexión intervertebral torácica en el test de distancia dedos-planta. Además, una mayor flexión intervertebral aumenta la presión intradiscal (Polga et al., 2004; Wilke et al., 1999) y el estrés compresivo y de cizalla (Callaghan \& McGill, 1995; McGill, 2002), disminuyendo el número de ciclos de carga necesarios para producir una lesión en los tejidos vertebrales (Callaghan \& McGill, 2001).

Una de las principales limitaciones de este estudio fue no haber realizado una comparación del morfotipo raquídeo de los deportistas con una población sedentaria de su misma edad, con el objetivo de establecer la influencia del entrenamiento sistematizado en el morfotipo raquídeo. No obstante, otros estudios si han realizado esta comparación. Pastor (2000) analizó la disposición sagital del raquis en flexión máxima del tronco en nadadores de categoría infantil, evidenciando un mayor porcentaje de morfotipos cifóticos respecto a la población escolarizada. En cuanto a la curva lumbar, los nadadores presentaban menor frecuencia de morfotipos cifóticos. Martínez (2004), sin embargo, encontró una menor frecuencia de morfotipos dorsales alterados en flexión máxima del tronco en gimnastas de rítmica, respecto a un grupo control. No obstante, en cuanto a la curva lumbar encontró una mayor frecuencia de morfotipos cifóticos en las gimnastas. Las diferencias entre ambos estudios podrían ser consecuencia de una importante diferencia en la extensibilidad isquiosural de los deportistas, ya que las gimnastas tenían una extensibilidad mayor que los nadadores y los grupos control.

El seguimiento de la evolución de la postura corporal en deportistas debería integrarse en la planificación del entrenamiento, ya que las curvas raquídeas cambian con el crecimiento (Widhe, 2002) y el entrenamiento sistematizado (Wojtys et al., 2000). Alricsson y Werner (2006), en esquiadores de fondo, encontraron un incremento significativo de la cifosis torácica en bipedestación tras un período de 5 años de entrenamiento.

Al valorar el morfotipo raquídeo en máxima flexión del tronco hay que tener en cuenta la extensibilidad de la musculatura isquiosural y la percepción de los movimientos pélvicos, ya que en ocasiones es el bloqueo coxofemoral y la mala utilización del ritmo lumbopélvico lo que produce una mayor flexión raquídea (Fisk \& Baigent, 1984; Stokes \& Abery, 1980).

El desarrollo de una adecuada extensibilidad isquiosural es muy importante puesto que varios estudios ponen de manifiesto la existencia 
de un alto porcentaje de deportistas con una extensibilidad reducida (Ferrer, 1998; López-Miñarro,Alacid, Ferragut, \& García, 2008b; LópezMiñarro, Ferragut, Alacid, Yuste, \& García, 2008c; Pastor, 2000; Wang, Whitney, Burdett, \& Janosky, 1993), debido principalmente a un volumen insuficiente de estiramientos, ya que diversos programas de estiramiento isquiosural han mostrado mejoras significativas con un volumen moderado de entrenamiento (Halbertsma \& Göeken, 1994; Rodríguez-García, Santonja, López-Miñarro, Sáinz de Baranda, \& Yuste, 2008b; Zakas, Galazoulas, Grammatikopoulou, \& Vergou, 2002).

Para mejorar la disposición raquídea en bipedestación, y especialmente en flexión del tronco, es preciso incorporara la planificación del entrenamiento de los deportistas, un programa de postura corporal. Puesto que la postura del raquis dorso-lumbar está íntimamente relacionada con la posición de la pelvis (Levine \& Whittle, 1996), yésta con la extensibilidad isquiosural en posiciones de flexión del tronco con rodillas extendidas (Gajdosik, Albert, \& Mitman, 1994; López-Miñarro y Alacid, 2009; López-Miñarro, Sáinz de Baranda, Rodríguez-García, \& Yuste, 2008e; López-Miñarro, Sáinz de Baranda, \& RodríguezGarcía, 2009; Rodríguez-García, López-Miñarro, Yuste, \& Sáinz de Baranda, 2008a), un adecuado trabajo de concienciación pélvica y un entrenamiento sistematizado de la extensibilidad, deberían ser incluidos en los programas de entrenamiento.

Es importante tener en cuenta que la flexión del tronco se efectúa de manera repetida en gestos de la vida cotidiana, así como en gestos deportivos. Detectar una mala disposición del raquis es esencial para implantar una precisa corrección (Santonja \& Martínez, 1995; Santonja et al., 2000). El análisis del morfotipo raquídeo en máxima flexión del tronco es necesario porque complementa la exploración en bipedestación, pudiendo detectar posibles patologías raquídeas y, por tanto, diseñar un protocolo de tratamiento o de prevención adecuado según se detecte (Martínez, 2004):

- Una curva aumentada en bipedestación con un claro incremento de la misma en posición de máxima flexión del tronco, lo que sugiere una estructuración de la misma.

- Un incremento de la curva dorsal en flexión máxima del tronco con una disposición angular normal en bipedestación, característico de la cifosis funcional, y que va a ser un factor que predispondrá a una disposición patológica en años posteriores en la posición de bipedestación.

- Un morfotipo cifótico lumbar en flexión máxima del tronco con una curva lumbar normal en bipedestación, así como en aquellos con hiperlordosis lumbar.

Además, ante deportistas que presentan estos morfotipos raquídeos hay que considerar la elección de ejercicios alternativos a los tradicionalmente realizados (Herrador, Latorre, \& Osorio, 2002), ya que con frecuencia en estos se adoptan posturas que inciden negativamente en el desarrollo del morfotipo raquídeo del deportista.

Las herramientas y test para el control y la cuantificación del entrenamiento no son la clave de una planificación exitosa, pero facilitan el proceso de elaboración, corrección y ajuste de las programaciones de entrenamiento realizadas (Alacid, 2008). La postura corporal de los deportistas también debe ser una variable a incluir en la planificación del entrenamiento deportivo.

Estudios previos han mostrado que las posturas y gestos técnicos específicos de cada deporte condicionan el morfotipo raquídeo de los deportistas. La mayor parte de los estudios realizados hasta el momento no han analizado la disposición sagital del raquis en flexión máxima del tronco, por lo que no se ha podido establecer la frecuencia de casos de cifosis funcional y actitud cifótica lumbar en estos deportistas. El presente estudio ha analizado una muestra de piragüistas varones, que se caracterizan por una posición de sedentación prolongada con las rodillas ligeramente flexionadas. Esta posición, mantenida frecuentemente, es probablemente un factor determinante en la frecuencia de casos de cifosis funcional y actitud cifótica lumbar. No obstante, puesto que sólo se han valorado a piragüistas varones, es preciso analizar si existen diferencias en función del género, así como comparar el morfotipo dinámico del raquis entre deportistas de diversas disciplinas.

\section{Conclusiones}

En la valoración de la disposición sagital del raquis en deportistas jóvenes es necesario complementar la exploración en bipedestación habitual con la realizada en flexión máxima del tronco con rodillas extendidas, pues existe una alta frecuencia de deportistas que presentan una cifosis funcional y/o actitud cifótica lumbar. Por ello, es preciso instaurar un programa de intervención postural para mejorar la disposición angular del raquis en flexión máxima del tronco, en la medida de lo posible.

\section{Referencias bibliográficas}

Alacid, F. (2008). Hoja de cálculo para la cuantificación del entrenamiento en piragüismo. Retos. Nuevas tendencias en Educación Física, Deporte y Recreación, 14, 54-58.

Alricsson, M. y Werner, S. (2006). Young elite cross-country skiers and low back pain- A 5-year study. Physical Therapy in Sport, 7, 181184.

Ashton-Miller, J.A. (2004). Thoracic hyperkyphosis in the young athlete: areview of the biomechanical issues. Current Sports Medicine Report, 3, 47-52.

Boldori, L., Da Soldá, M. y Marelli, A. (1999). Anomalies of the trunk. An analysis of their prevalence in young athletes. Minerva Pediatrica, 51, 259-264.

Briggs, A. M., Van Dieën, J. H., Wrigley, T. V., Greig, A. M., Phillips, B., Lo, S. K. y Bennell K.L. (2007). Thoracic kyphosis affects spinal loads and trunk muscle force. Physical Therapy, 87, 595-607.

Callaghan, J. P y McGill, S. M. (2001). Low back joint loading and kinematics during standing and unsupported sitting. Ergonomics, 44, 280-294.

Callaghan, J. P. y McGill, S. M. (1995). Muscle activity and low back loads under external shear and compressive loading. Spine, 20, $992-$ 998.

Domisse, G. F. (1990). The vulnerable, rapidly growing thoracic spine of the adolescent. South African Medical Journal, 78, 211-213.

Esola, M. A., McClure, P. W., Fitzgerald, G. K. y Siegler, S. (1996). Analysis of lumbar spine and hip motion during forward bending in subjects with and without a history of low back pain. Spine, 21, 71-78.

Ferrer, V. (1998). Repercusiones de la cortedad isquiosural sobre la pelvis y el raquis lumbar. Tesis Doctoral. Universidad de Murcia.

Fisk, J. W., Baigent, M, L. y Hill, P. D. (1984). Scheuermann's disease. Clinical and radiological survey of 17 and 18 years old. American Journal of Physical Medicine, 63, 18-30.

Gajdosik, R. L., Albert, C. R. y Mitman, J. J. (1994). Influence of hamstring length on the standing position and flexion range of motion of the pelvic angle, lumbar angle, and thoracic angle. Journal of Orthopaedic and Sports Physical Therapy, 20, 213-219.

Gómez, S. (2007). Estudio sagital del raquis en bailarinas de danza clásica y danza española. Tesis Doctoral, Universidad de Murcia.

Halbertsma, J. P. K. y Göeken, L. N. H. (1994). Stretching exercises: Effect on passive extensibility and stiffness in short hamstrings of healthy subjects. Archives of Physical Medicine and Rehabilitation, 75, 976-981.

Herrador, J.A., Latorre, P. y Osorio, M. M. (2002). Accidentes durante la práctica de actividades físico-deportivo-recreativas. Retos. Nuevas tendencias en Educación Física, Deporte y Recreación, 2, 21-29.

Howell, D. W. (1984). Musculoskeletal profile and incidence of musculoskeletal injuries in lightweight women rowers. American Journal of Sports Medicine, 12, 278-281.

Kums, T., Ereline, J., Gapeyeva, H., Pääsuke, M. y Vain, A. (2007). Spinal curvature and trunk muscle tone in rhythmic gymnasts and untrained girls. Journal of Back and Musculoskeletal Rehabilitation, 20, 87-95.

López, N., Alburquerque, F., Quintana, E., Domínguez, R., Rubens, J. y Calvo, J. I. (2005). Evaluación y análisis del morfotipo raquídeo del futbolista juvenil y amateur. Fisioterapia, 27, 192-200. 
López-Miñarro, P. A., Alacid, F., Ferragut, C. y García, A. (2008a). Valoración y comparación de la disposición sagital del raquis entre canoistas y kayakistas de categoría infantil. Cultura, Ciencia y Deporte, 9, 171-176.

López-Miñarro, P. A., Alacid, F., Ferragut, C. y García, A. (2008b). Valoración y comparación de la extensibilidad isquiosural entre kayakistas y canoistas de categoría infantil. Motricidad. European Journal of Human Movement, 20, 97-111.

López-Miñarro, P. A., Ferragut, C., Alacid, F., Yuste, J. L. y García, A. (2008c). Validez de los test dedos-planta y dedos-suelo para la valoración de la extensibilidad isquiosural en piragüistas de categoría infantil. APUNTS Medicina Deportiva, 157, 24-29.

López-Miñarro, P. A., Rodríguez, P. L., Santonja, F. M. y Yuste, J. L. (2008d). Posture of thoracic spine during triceps-pushdown exercise. Science \& Sports, 23, 183-185.

López-Miñarro, P. A., Rodríguez, P. L., Santonja, F., Yuste, J. L. y García,A. (2007a). Sagittal spinal curvatures in recreational weight lifters. Archivos de Medicina del Deporte, 122, 435-441.

López-Miñarro, P. A., Sáinz de Baranda, P., Rodríguez-García, P. L. y Yuste, J. L. (2008e). Comparison between the sit-and-reach test and V sit-and-reach test in young adults. Gazzetta Medica Italiana, $167,135-142$

López-Miñarro, P. A., Sáinz de Baranda, P. y Rodríguez-García, P. L. (2009). A comparison of the sit-and-reach test and the back-saver sit-and-reach test in university students. Journal of Sports Science and Medicine, 8, 116-122.

López-Miñarro, P. A., Yuste, J. L., Rodríguez, P. L., Santonja, F., Sáinz de Baranda P. y García, A. (2007b). Sagittal spinal curvatures of thoracic and lumbar spine during the standing bilateral curl bar exercise. Cultura, Ciencia y Deporte, 7, 19-24.

López-Miñarro, P.A. y Alacid, F. (2009). Influence of hamstring muscle extensibility on spinal curvatures in young athletes. Science \& Sports. doi:10.1016/j.scispo.2009.10.004

Martínez, F. M. (2004). Disposición del raquis en el plano sagital y extensibilidad isquiosural en gimnasia rítmica deportiva. Tesis Doctoral. Universidad de Murcia.

McGill, S. M. (2002). Low back disorders. Evidence-Based prevention and rehabilitation. Champaign: Human Kinetics.

Miñarro, P. A., Andújar, P. S., García, P. L. y Toro, E. O. (2007). A comparison of the spine posture among several sit-and-reach test protocols. Journal of Science and Medicine in Sport, 10, 456-462.

Murray, P. M., Weinstein, S. L. y Spratt, K. F. (1993). The natural history and long-term follow-up of Scheüermann kyphosis. Journal of Bone and Joint Surgery, 75, 236-248.

Nilsson, C., Wykman,A. y Leanderson, J.(1993). Spinal Sagittal mobility and joint laxity in young ballet dancers. Knee Surgery, Sports Traumatology, Arthroscopy, 1, 206-208.

Ohlén, G., Wredmark, T. y Spandfort, E. (1989). Spinal sagittal configuration and mobility related to low-back pain in the female gymnast. Spine, 14, 847-850.

Pastor, A. (2000). Estudio del morfotipo sagital de la columna y de la extensibilidad de la musculatura isquiosural de jóvenes nadadores de élite Españoles. Tesis Doctoral. Universidad de Murcia.

Polga, D. J., Beaubien, B. P., Kallemeier, P. M., Schellhas, K. P., Lee, W. D., Buttermann, G. R. y Wood, K. B. (2004). Measurement of in vivo intradiscal pressure in healthy thoracic intervertebral discs. Spine, 29, 1320-1324.

Rajabi, R., Doherty, P., Goodarzi, M. y Hemayattalab, R. (2008) Comparison of thoracic kyphosis in two groups of elite GrecoRoman and free style wrestlers and a group of non-athletic subjects. British Journal of Sports Medicine, 42, 229-232.

Rodríguez, P. L. (1998). Educación Física y salud del escolar: Programa para la mejora de la extensibilidad isquiosural y del raquis en el plano sagital. Tesis Doctoral, Universidad de Granada.

Rodríguez-García, P. L., López-Miñarro, P. A., Yuste, J. L. y Sáinz de Baranda, P. (2008a). Comparison of hamstring criterion-related validity, sagittal spinal curvatures, pelvic tilt, and score between the sit-and-reach and toe-touch tests in athletes. Medicina dello Sport, 61, 11-20.

Rodríguez-García, P. L., Santonja, F., López-Miñarro, P. A., Sáinz de Baranda, P. y Yuste, J. L. (2008b). Effect of physical education stretching programme on sit-and-reach score in schoolchildren. Science \& Sports, 23, 170-175.

Sáinz de Baranda, P., Ferrer, V., Martínez, L., Santonja, F., Rodríguez, P. L., Andújar, P. Carrión, M. y García, M. J. (2001). Morfotipo del futbolista profesional. Actas del segundo Congreso Internacional de Educación Física y Diversidad (pp. 293-295). Consejería de Educación y Universidades: Murcia.

Santonja, F. (1993). Exploración clínica y radiológica del raquis sagital. Sus correlaciones (premio SOCUMOT-91). Murcia: Secretariado de Publicaciones e intercambio científico.

Santonja, F., Rodríguez, P. L., Sáinz de Baranda, Py López-Miñarro, P. A. (2004). Papel del profesor de educación física ante las desalineaciones del raquis. Selección, 13, 5-17.

Santonja, F. y Martínez, I. (1995). Raquis y deporte ¿cuál sí y cuándo? Selección, 4, 28-38.

Santonja, F. (1996). Las desviaciones sagitales del raquis y su relación con la práctica deportiva. En: Ferrer V, Martínez L, Santonja F. Escolar, Medicina y Deporte. Diputación Provincial de Albacete; 251-268.

Santonja, F.; Pastor,A.; Serna, L. (2000). Valoración radiográfica de las desalineaciones sagitales del raquis. Selección, 9, 216-229.

Serna, L., Santonja, F. y Pastor,A. (1996). Exploración clínica del plano sagital del raquis. Selección, 5, 88-102.

Solomonow, M. (2004). Ligaments: a source of work-related musculoskeletal disorders. Journal of Electromyographic and Kinesiology, 14, 49-60.

Somhegyi, A. y Ratko, I. (1993). Hamstring Tightness and Scheuermann's Disease. American Journal of Physical Medicine an Rehabilitation, 72, 44 .

Stagnara, P. (1987). Deformaciones del raquis. Escoliosis, cifosis, lordosis. Barcelona: Masson.

Stokes, I. A. F. y Abery, J. M. (1980). Influence of the hamstring muscles on lumbar spine curvature in sitting. Spine, 5, 525-528.

Swärd, L. (1992). The thoracolumbar spine in young elite athletes. Current concepts on the effects of physical training. Sports Medicine, 13, 357-364.

Uetake, T., Ohsuki, F., Tanaka, H. y Shindo, M. (1998). The vertebral curvature of sportsmen. Journal of Sports Sciences, 16, 621-628.

Ventura, N. (1986). Las desviaciones de la columna vertebral. Medicina Integral, 8, 461-467.

Wang, S. S., Whitney, S. L., Burdett, R. G. y Janosky, J. E. (1993). Lower extremity muscular flexibility in long distancerunners. Journal of Orthopaedic and Sports Physical Therapy, 17, 102-107.

Widhe, T. (2001). Spine: posture, mobility and pain. A longitudinal study from childhood to adolescence. European Spine Journal, 10, 118-123.

Wilke, H. J., Neef, P., Caimi, M., Hoogland, T. y Claes, L. E. (1999). New in vivo measurements of pressures in the intervertebral disc in daily life. Spine, 24, 755-762.

Wodecki, P., Guigui, P., Hanotel, M. C., Cardinne, L., Deburge, A. (2002). Sagittal alignment of the spine: comparison between soccer players and subjects without sports activities. Revue de Chirurgie Orthopédique et Réparatrice de L'appareil Motear, 88, 328-336.

Wojtys, E., Ashton-Miller, J., Huston, L. y Moga, P. (2000). The association between athletic training time and the sagittal curvature of the immature spine. American Journal of Sports Medicine, 17, 490-498.

Zakas, A., Galazoulas, C., Grammatikopoulou, M. G. y Vergou, A. (2002). Effects of stretching exercise during strength training in prepubertal, pubertal and adolescent boys. Journal of Bodywork and Movement Therapies, 6, 170-176. 